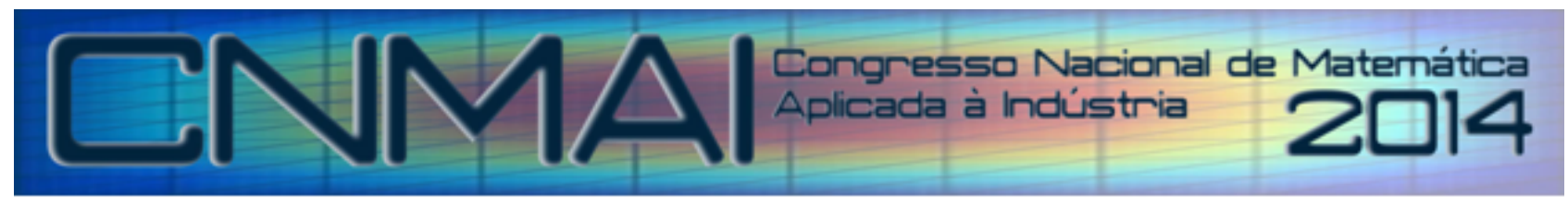

18 a 21 de novembro de 2014, Caldas Novas - Goiás

\title{
CONSERVAÇÃO DE VOLUME EM TÉCNICAS DE TRATAMENTO DE INTERFACE EM MÉTODOS ISPH
}

\author{
Douglas Farias Cordeiro, cordeiro@ufg.br ${ }^{1}$ \\ Ana Teresa Chaves Leite, aninhagta . chaves@gmail . com ${ }^{2}$ \\ Ciro José Almeida Macedo, ciro.macedo@ifg.edu. br ${ }^{2}$ \\ Marcos Aurélio Batista, marcos@catalao.ufg.br ${ }^{3}$ \\ ${ }^{1}$ Universidade Federal de Goiás - Faculdade de Informação e Comunicação \\ Campus Samambaia, Goiânia - GO, 74.001-190. \\ ${ }^{2}$ Instituto Federal de Educação, Ciência e Tecnologia de Goiás \\ Campus Cidade de Goiás, Quartel do XX, Praça Brasil Ramos Caidado, Goiás - GO, 76.600-000. \\ ${ }^{3}$ Universidade Federal de Goiás - Departamento de Ciência da Computação \\ Av. Dr. Lamartine P. Avelar, 1120, Catalão - GO, 75.704-020.
}

\begin{abstract}
Resumo. O método SPH é um método de solução numérica baseado na utilização de partículas e empregado em problemas de dinâmica de fluidos. Entre as principais estratégias de aplicação do método SPH, o ISPH se destaca como uma das alternativas que apresenta melhor acurácia e estabilidade nos resultados. Entretanto, quando aplicado como solução de escoamentos que possuem valores de Reynolds altos, o ISPH apresenta problemas de aglomeração de partículas, sendo necessário a utilização de técnicas auxiliares para a correção das posições das partículas, o que acaba por alterar as linhas de correntes naturais. Essa característica provoca problemas na simulação de escoamentos bifásicos, principalmente no que se refere à disposição da interface entre fluidos. Para prevenir isso, foi introduzida em Cordeiro et al. (2013) uma técnica de tratamento de interface baseada em Level Set, a qual apresenta resultados consideráveis, porém com algumas variações de volume durante as simulações, o que pode representar um problema em determinados tipos de simulações. Neste sentido, é apresentado aqui um aperfeiçoamento da técnica de tratamento de interface, através da incorporação de rotinas que previnem tais variações de volume.
\end{abstract}

Palavras-chave: sph, métodos numéricos, conservação de volume, escoamentos bifásicos

\section{INTRODUÇÃO}

Em 1977 Gingold e Monaghan (1977), e independentemente Lucy (1977), introduziram um método numérico a ser aplicado em soluções de problemas astrofísicos em espaços tridimensionais. O método, denominado de SPH (Smoothed Particle Hydrodynamics), trata-se de um método puramente lagrangeano, onde o estado de um sistema é representado por um conjunto finito de partículas, as quais possuem propriedades como volume, massa, densidade e velocidade, e se movem de acordo com as equações governantes de conservação. A dinâmica do sistema é aproximada através das interações entre as partículas, as quais estão relacionadas à utilização de uma função núcleo, também conhecida por função suave.

A definição original do SPH o caracteriza como um método adequado para ser utilizado em processos de simulação de escoamentos de fluidos compressíveis. Entretanto, sua aplicação como solução de escoamentos incompressíveis não apresentava resultados satisfatórios. Neste sentido, a busca por soluções numéricas para este tipo de problema levou ao aperfeiçoamento e adequação do método, e através de uma estratégia que considera os fluidos incompressíveis como quase-compressíveis, foi introduzido por Monaghan (1994) um novo método, denominado WCSPH (Weakly Compressible Smoothed Particle Hydrodynamics). No WCSPH a pressão é resolvida através da utilização de uma equação de estado, que baseia-se principalmente na variação da densidade de partículas e na velocidade do som no meio. Apesar de proporcionar bons resultados para algumas classes de problemas e a vantagem de ser um método completamente livre de malhas, esta abordagem apresentou alguns problemas e limitações, associados principalmente ao cálculo da pressão e a necessidade de passos de tempos pequenos. 
Com o objetivo de resolver as limitações existentes no WCSPH, Cummins e M. (1999), utilizando como base o método da projeção, propuseram uma nova abordagem para o procedimento de cálculo da pressão no SPH. De acordo com a nova abordagem, a resolução da pressão é realizada através do uso de uma equação de Poisson, e além disso, o valor da densidade é considerado constante durante toda a simulação. Esta nova abordagem foi denominada de ISPH (Incompressible Smoothed Particle Hydrodynamics), e proporcionou uma considerável melhora nos resultados obtidos, além de permitir a utilização de passos de tempo maiores e a eliminação da dependência de definição de um valor para a velocidade do som. Entretanto, a nova estratégia apresentou problemas em relação à aplicação para a solução de escoamentos com elevados números de Reynolds, onde foram observados a formação de agrupamentos de partículas, devido ao alto movimento inercial relacionado.

As limitações do ISPH, especialmente no que diz respeito ao problema de agrupamento de partículas, levou à concepção de modelos alternativos, os quais propuseram, principalmente, uma variação na construção da equação de Poisson, levando-se em conta informações referentes à variação da densidade, a qual, nestes modelos, não é considerada fixa Shao e Lo (2003); Hu e Adams (1995). Além disso, também foram propostas técnicas de tratamento da distribuição de partículas, sem alteração do modelo original do ISPH. Entre estas técnicas, a que mostrou-se mais interessante foi a inserção de um deslocamento artificial, baseado na distribuição das partículas, com consecutiva correção das variáveis hidrodinâmicas Xu et al. (2009).

Apesar do método ISPH proporcionar bons resultados para a solução de escoamentos newtonianos monofásicos, sua aplicação para escoamentos multifásicos ainda possui alguns obstáculos, principalmente no que se refere à manutenção da interface entre fluidos, devido a problemas relacionados à interpenetração de partículas entre as fases. Neste sentido, foi proposta por Cordeiro et al. (2013) uma técnica de correção da interface baseada na utilização de uma função Level Set. Embora esta técnica proporcione resultados consideráveis, ela apresenta a desvantagem de não garantir a conservação de volume ao longo da simulação, o que pode acabar por prejudicar a acurácia do sistema. Diante disso, neste trabalho é proposto um aperfeiçoamento desta técnica, com base em uma redistribuição de massa, de modo a garantir a conservação do volume ao longo das simulações.

\section{EQUAÇÕES DE CONSERVAÇÃO}

Os métodos ISPH podem ser aplicados como forma de aproximação para problemas regidos pelas Equações de Navier-Stokes, as quais podem ser descritas, em sua forma Lagrangeana, como:

$$
\begin{array}{r}
\nabla \cdot \mathbf{v}=0, \\
\frac{D \mathbf{v}}{D t}=-\frac{1}{\rho} \nabla p+\frac{\mu}{\rho} \nabla^{2} \mathbf{v}+\mathbf{F} .
\end{array}
$$

sendo $\rho$ a densidade, $p$ a pressão, $\mu$ a viscosidade dinâmica, $\mathbf{v}$ o vetor velocidade e $\mathbf{F}$ as forças externas presentes no problema.

\section{O MÉTODO ISPH}

\subsection{Formulação SPH}

De um modo geral, os métodos baseiam-se no fato de uma uma função $f$ qualquer pode ser representada em uma forma integral, dita rigorosa ou exata, dada por:

$$
f(\mathbf{x})=\int f\left(\mathbf{x}^{\prime}\right) \delta\left(\mathbf{x}-\mathbf{x}^{\prime}\right) d \mathbf{x}^{\prime},
$$

sendo $\delta\left(\mathbf{x}-\mathbf{x}^{\prime}\right)$ o Delta de Dirac. Sabe-se que não é possível definir uma função capaz de satisfazer as propriedades de $\delta\left(\mathbf{x}-\mathbf{x}^{\prime}\right)$. Uma alternativa para resolver este problema é utilizar uma aproximação por uma função núcleo $W$, que pode ser descrita como:

$$
f(\mathbf{x}) \approx \int f\left(\mathbf{x}^{\prime}\right) W\left(\mathbf{x}-\mathbf{x}^{\prime}\right) d \mathbf{x}^{\prime},
$$

e discretizada como:

$$
f(\mathbf{x}) \approx \sum_{j \in \Omega_{i}} \frac{m_{j}}{\rho_{j}} f\left(\mathbf{x}_{j}\right) W_{i j},
$$

sendo $m_{j}$ a massa da partícula e $W_{i j}=W\left(\mathbf{x}_{i}-\mathbf{x}_{j}, h\right)$. Tal discretização parte do fato de que a integral de uma determinada função aplicada a um ponto do domínio $\Theta$, pode ser discretizada como o somatório sobre as partículas vizinhas, as quais 
encontram-se no sub-espaço $\Omega_{i}$ de $\Theta$, tal que $\Omega_{i}=\mathbf{x}_{j},\left|\mathbf{x}_{i}-\mathbf{x}_{j}\right| \leq \kappa h$, com $h$ referindo-se ao comprimento do núcleo e $\kappa$ a constante que determina a área não nula da função núcleo. Além disso, é importante destacar que o volume infinitesimal $d \mathbf{x}$ é aproximado pelo volume ocupado por uma partícula, representado por $\delta V$, o que equivale a razão entre massa e densidade da partícula.

De maneira similar ao apresentado anteriormente, é possível se obter os operadores SPH para o cálculo de derivadas, como pode ser visto em Liu e Liu (2003). É importante destacar que existem diferentes tipos de operadores. Em Petronetto (2008) é apresentada uma análise sobre operadores SPH.

Neste trabalho, será utilizado o seguinte operador gradiente SPH:

$$
\nabla \phi_{i}=\sum_{j} \frac{m_{j}}{\rho_{j}} \phi_{j i} \nabla W_{i j}
$$

o qual é similar ao operador divergente, os quais são utilizados para aproximação do gradiente de pressão e do divergente de velocidade.

Por outro lado, o termo viscoso, presente na equação 2 pode ser aproximado por:

$$
\left(\frac{\mu}{\rho} \nabla^{2} \mathbf{v}\right)_{i}=\sum_{j} \frac{m_{j}\left(\mu_{i}+\mu_{j}\right) \mathbf{x}_{i j} \cdot \nabla W_{i j}}{\rho_{j} \mathbf{x}_{i j}^{2}} \mathbf{v}_{i j} .
$$

Além disso, é importante destacar que o laplaciano da pressão utiliza um operador específico introduzido em Cleary e Monaghan (1999), o qual é mais adequado para escoamentos multifásicos:

$$
\left(\nabla \cdot\left(\frac{1}{\rho} \nabla p\right)\right)_{i}=\sum_{j} \frac{m_{j}}{\rho_{j}}\left(\frac{4}{\rho_{i}+\rho_{j}}\right) p_{i j} \frac{\mathbf{x}_{i j}}{\mathbf{x}_{i j}^{2}} \nabla_{i} W_{i j}
$$

Os testes realizados neste artigo utilizam uma função núcleo de quinta ordem, introduzida por Morris (1996) e determinada como:

$$
\mathcal{W}(R)=\alpha_{d} \begin{cases}(3-R)^{5}-6(2-R)^{5}+15(1-R)^{5} & , 0 \leq R<1 \\ (3-R)^{5}-6(2-R)^{5} & , 1 \leq R<2 \\ (3-R)^{5} & , 2 \leq R \leq 3 \\ 0 & , R>3\end{cases}
$$

com $\alpha_{1}=120 / h, \alpha_{2}=7 /\left(478 \pi h^{2}\right)$ e $\alpha_{3}=3 /\left(359 \pi h^{3}\right)$, e considerando que $\mathcal{W}(R)=W\left(\mathbf{x}_{i}-\mathbf{x}_{j}, h\right)$ e $R=\frac{\left\|\mathbf{x}_{i}-\mathbf{x}_{j}\right\|}{h}$.

\subsection{Estratégia de solução do ISPH}

Uma das características do ISPH é ter o valor da densidade definido como constante, sendo associado às partículas no passo inicial de simulação, o que não ocorre no WCSPH. Esta estratégia garante a não violação da condição de incompressibilidade, representada na equação 2. A partir disso, o método utiliza a equação de conservação da quantidade de movimento (equação 2) para obter a dinâmica do sistema. Para tal, a primeira tarefa, em cada passo de simulação, é definir uma posição intermediária $\mathbf{x}^{*}$ :

$$
\mathbf{x}_{i}^{*}=\mathbf{x}_{i}^{n}+\Delta t \mathbf{v}_{i}^{n}
$$

e, através disso, um campo de velocidades intermediário $\mathbf{v}^{*}$, o qual refere-se à um campo de velocidade livre de divergência, sendo este calculado com base na equação de quantidade de movimento sem a contribuição do gradiente de pressão, tal que:

$$
\mathbf{v}_{i}^{*}=\mathbf{v}_{i}^{n}+\left(\mu \nabla^{2} \mathbf{v}_{i}^{n}\right) \Delta t
$$

A pressão, no tempo $n+1$, é então calculada através da seguinte equação de Poisson:

$$
\nabla \cdot\left(\frac{1}{\rho} \nabla p\right)_{i}=\frac{1}{\Delta t} \nabla \cdot \mathbf{v}_{i}^{*}
$$

Utilizando-se os valores de pressão calculados, é possível corrigir o campo de velocidade livre de divergência, no tempo $n+1$, onde: 


$$
\mathbf{v}_{i}^{n+1}=\mathbf{v}_{i}^{*}-\left(\frac{1}{\rho} \nabla p^{n+1}\right) \Delta t
$$

e, finalmente, calcular a nova posição das partículas:

$$
\mathbf{x}_{i}^{n+1}=\mathbf{x}_{i}^{n}+\frac{\Delta t}{2}\left(\mathbf{v}_{i}^{n+1}+\mathbf{v}_{i}^{n}\right)
$$

Uma maneira de se impor condições de contorno em soluções através do ISPH é através da utilização de partículas fantasmas (Liu e Liu (2003)). Nos problemas abordados neste trabalho são consideradas condições de contorno de Dirichlet para a velocidade e de Neumann para a pressão.

\subsection{Correção de posição no ISPH}

Embora o ISPH apresente bons resultados e seja consideravelmente mais estável que o WCSPH, as características do próprio método fazem que as partículas sigam continuamente as linhas de corrente do escoamento, o que pode acabar gerando regiões com aglomerados ou de baixa densidade de partículas. Esse fenômeno é um problema crítico em soluções através do ISPH, uma vez que pode ter consequências indesejáveis, tais como o mal condicionado da matriz referente à equação de Poisson (12). Na Fig. 1- esquerda é apresentado um passo de simulação do experimento clássico vórtice simples, onde é possível notar claramente a formação de agloramerados e regiões vazias. A fim de corrigir este problema, $\mathrm{Xu}$ et al. (2009) introduziram uma técnica de correção de posição, a qual consiste na aplicação de um deslocamento artificial das partículas e na posterior correção das variáveis hidrodinâmicas.
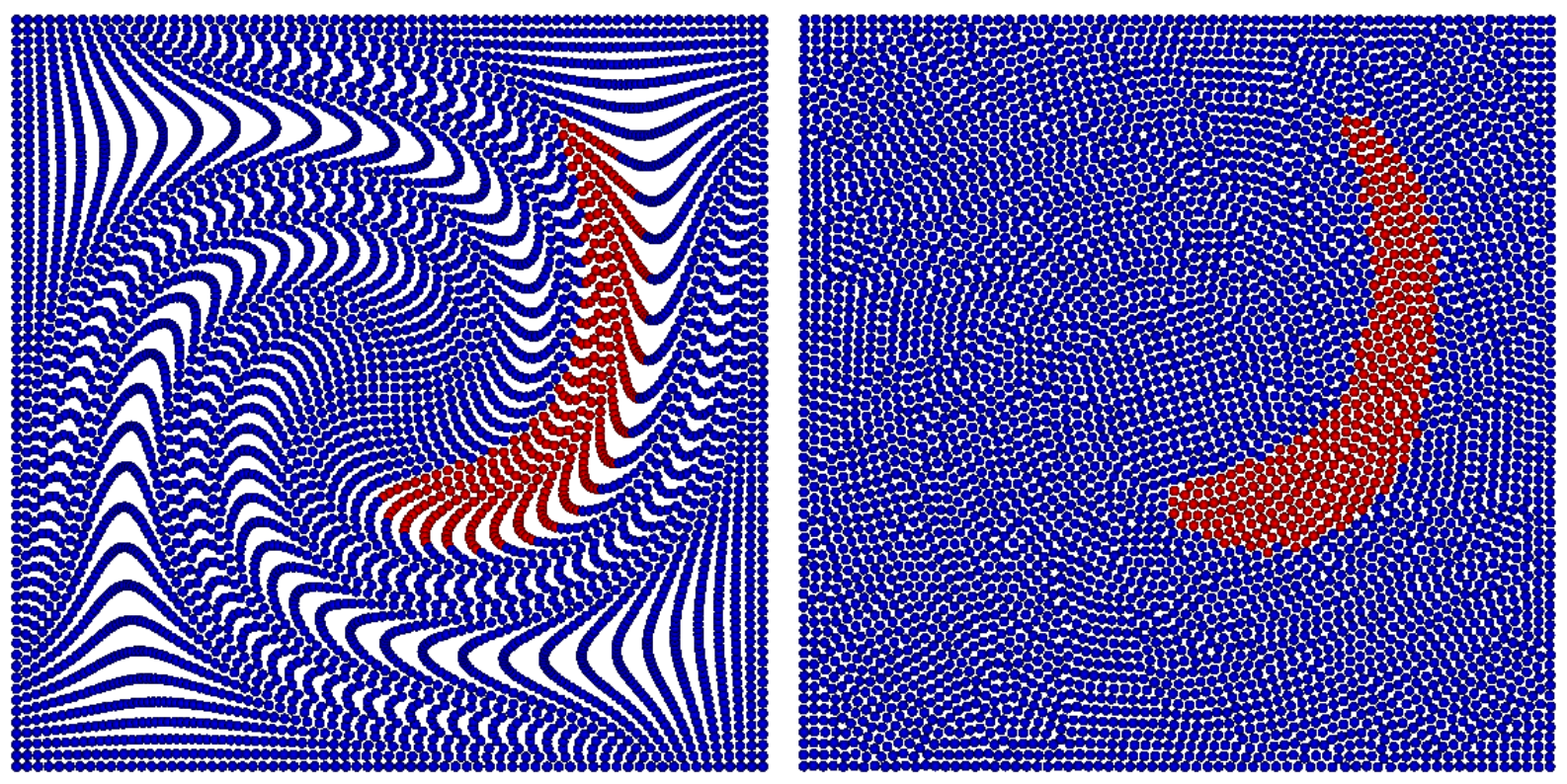

Figura 1. Problema de agrupamento de partículas em altos Reynolds usando o ISPH.

Para aplicar a técnica proposta por Xu et al. (2009), após a obtenção de $\mathbf{x}_{i}^{n+1}$ através da aproximação ISPH (Equação 14), é calculado o deslocamento artificial:

$$
\delta \mathbf{x}_{i}=C \alpha X_{i}
$$

onde $C$ é uma constante entre 0.01 e $0.1, \alpha$ é a magnitude do deslocamento, tal que $\alpha=\mathbf{V}_{\max } \Delta t$, tendo $\mathbf{V}_{\max }$ como a velocidade máxima do sistema e $X_{i}$ como o vetor deslocamento, sendo este calculado como:

$$
X_{i}=\sum_{j} \frac{\overline{\mathbf{x}}_{i}^{2}}{\mathbf{x}_{i j}^{2}} \mathbf{n}_{i j}, \operatorname{com} \overline{\mathbf{x}}_{i}=\frac{1}{M_{i}} \sum_{j} \mathbf{x}_{i j},
$$

sendo $M_{i}$ a quantidade de vizinhos da partícula $i$. Neste sentido, efetua-se a correção das variáveis hidrodinâmicas (velocidade e pressão) através de uma expansão de primeira ordem em Série de Taylor, tal que:

$$
\phi_{i^{\prime}}=\phi_{i}+\delta \mathbf{x}_{i i^{\prime}} \cdot(\nabla \phi)_{i}
$$

sendo que $\phi$ refere-se à variável a ser corrigida e $\delta \mathbf{x}_{i i^{\prime}}$ trata-se do vetor distância entre a posição antiga $\mathbf{x}_{i}$ e nova posição $\mathbf{x}_{i^{\prime}}$. O erro da correção é da ordem de $\mathcal{O}\left(\delta r_{i i^{\prime}}^{2}\right)$. A Fig. 1 - direita mostra o resultado deste procedimento. 


\section{CORREÇÃO DE INTERFACE BASEADA EM SÉRIE DE TAYLOR}

O método ISPH em conjunto com a técnica de correção de posição de Xu et al. (2009) pode apresentar problemas quando aplicado em escoamentos multifásicos, uma vez que o deslocamento artificial realizado pode acabar por provocar a interpenetração de partículas através da interface entre fluidos. Este problema torna-se ainda maior quando a interface entre fluidos está localizada em uma região que possui um alto movimento inercial, e consequentemente com grande chance de formação de áreas de aglomeramento ou áreas de baixa densidade de partículas, as quais serão tratadas através de sucessivos deslocamentos artificiais, sendo provável que partículas próximas à interface sejam deslocadas para o interior do outro fluido. Para minimizar este problema, foi proposta por Cordeiro et al. (2013) uma técnica de tratamento de interface baseada no uso de uma função level-set.

De um modo geral, a técnica determina uma função level-set em que os zeros encontrem-se exatamente sobre a interface entre fluidos. Além disso, a função associa valores positivos a uma fase, e valores negativos a outra fase. Para tanto, é utilizada uma função distância com sinal. As informações referentes à localização da interface podem ser tanto consideradas como entrada do problema, ou também podem ser obtidas através da utilização de técnicas específicas de detecção de interface. A partir desta informação, é então possível construir uma função level-set sobre o conjunto de partículas de fluido do sistema, tal que:

$$
\phi_{i}=\left(x_{i}-x_{b}\right) \mathbf{n}_{x}+\left(y_{i}-y_{b}\right) \mathbf{n}_{y}
$$

sendo que $\mathbf{x}_{i}=\left(x_{i}, y_{i}\right), \mathbf{x}_{b}=\left(x_{b}, y_{b}\right)$ é a partícula de borda mais próxima à partícula $i$, e $\mathbf{n}=\left(\mathbf{n}_{x}, \mathbf{n}_{y}\right)$ refere-se à normal unitária da partícula de borda $b$.

Sabe-se que em cada passo de simulação as posições das partículas são alteradas, logo tem-se que a interface entre fluidos continuamente modificada. Diante disso, a função level-set representa apenas a configuração inicial da interface, sendo então necessário sua constante atualização, com base na dinâmica do sistema. Para realizar tal correção pode ser utilizada uma estratégia à utilizada por Xu et al. (2009) na correção de partículas hidrodinâmicas. Neste sentido, a função $\phi$ pode então ser corrigida da seguinte maneira:

$$
\phi_{i}^{\prime}=\phi_{i}+\delta \mathbf{x}_{i i^{\prime}} \cdot(\nabla \phi)_{i}
$$

sendo que $i^{\prime}$ refere à partícula $i$ em sua posição corrigida, $\delta \mathbf{x}_{i i^{\prime}}$ ao deslocamento artificial aplicado à partícula $i$. Além disso, é importante destacar que o termo $\nabla \phi$ pode ser obtido através de uma aproximação SPH:

$$
(\nabla \phi)_{i}=\sum \frac{m_{j}}{\rho_{j}}\left(\phi_{j}-\phi_{i}\right) \nabla_{i} W_{i j}
$$

A partir dos valores da função $\phi$ é possível realizar uma avaliação em relação às posições das partículas de fluido em referência à interface entre fluidos. Para facilitar a compreensão do processo, sejam considerado um escoamento bifásico com dois fluidos A e B, de tal modo que para o fluido A são associados valores positivos e para o fluido B valores negativos. A partir disso, é realizado uma avaliação sobre todas as partículas de fluido, caso seja detectado que uma partícula de fluido A tenha valor de $\phi$ negativo, pode-se concluir que esta encontra-se fora de sua fase. O mesmo teste é realizado com as partículas do fluido B. Com base nisso, ao se detectar uma partícula fora de sua fase, suas propriedades alteradas, e esta passa a pertencer à outra fase.

Para exemplificar o problema da interpenetração de partículas no ISPH foi realizado um teste numérico com um campo de velocidades prescrito e fixo dado por:

$$
\begin{aligned}
& u(x, y, t)=-\cos (2 \pi x) \sin (2 \pi y) \\
& v(x, y, t)=\sin (2 \pi x) \cos (2 \pi y)
\end{aligned}
$$

onde é considerado um domínio bidimensional $[0,1] \times[0,1]$ e condições de contorno periódicas.

Para efeitos de validação, as partículas de fluidos são integradas temporalmente durante um certo tempo t/2. Após isso, o campo de velocidades é invertido, e as partículas são integradas até um tempo $t$. É importante destacar que a quantidade de passos de integração de 0 até $t / 2$ e de $t / 2$ até $t$ é equivalente. Neste sentido, espera-se que ao final da simulação a configuração inicial seja recuperada. Neste sentido, foi realizado um teste sobre a configuração representada na Fig. 3(a-d), onde em uma das fases as partículas encontram-se posicionadas dentro de um círculo de centro em $(0.5,0.5)$ e raio $r=0.4$, representadas na cor vermelha. Para a integração temporal foi utilizado um método Runge-Kutta de ordem 4, a fim de minimizar possíveis erros numéricos. Ao final da simulação a configuração inicial da interface é recuperada, tendo apenas os erros causados pelo método de integração, os quais são praticamente imperceptíveis.

Por outro lado, é importante considerar que ao se utilizar o método ISPH, situações em que ocorrem regiões com aglomeração ou vazios podem ser prejudiciais à estabilidade do sistema, sendo então necessária a utilização de técnicas 
de correção. Neste sentido, foi realizado o mesmo teste, porém aplicando a correção de posição proposta por Xu et al. (2009). A Fig. 3(e-h) apresenta a evolução deste problema, onde é possível notar que apesar da boa distribuição de partículas, a interface não foi bem recuperada (Fig. 2).

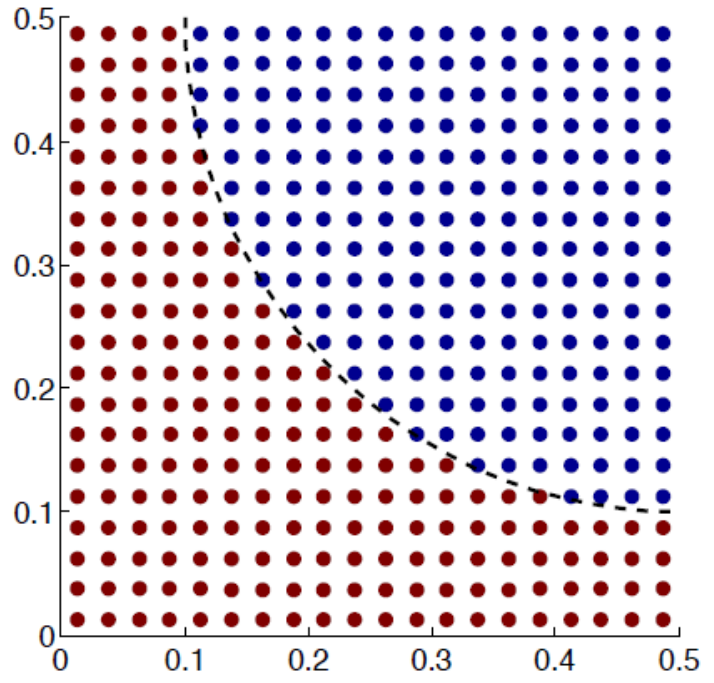

(a)

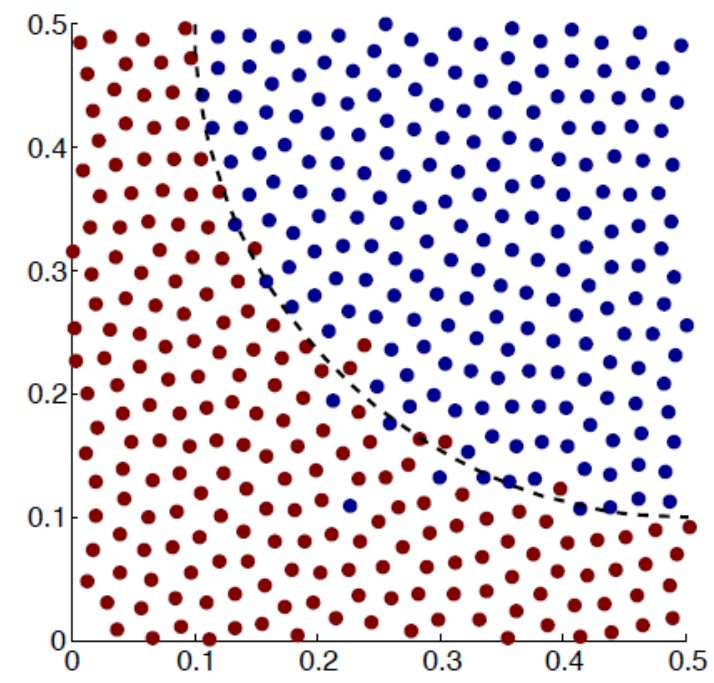

(b)

Figura 2. Detalhe da configuração final obtida: (a) sem correção de Xu et al. (2009), e (b) usando correção de Xu et al. (2009).

Finalmente, foi realizado um teste com a aplicação da correção da posição das partículas e utilização da técnica de correção de interface, onde foi utilizada uma função suave associada a cada partícula como:

$$
\Phi(x, y)=\sqrt{(x-0.5)^{2}+(y-0.5)^{2}}-0.4 .
$$

O campo $\Phi$ é negativo no interior do círculo, positivo no exterior, e permite uma boa definição da interface. Considerando o fato de que este campo é suave, aplica-se então, em cada passo de simulação, uma correção baseada em expansão em série de Taylor de primeira ordem:

$$
\Phi_{i^{\prime}}=\Phi_{i}+\delta \mathbf{x}_{i i^{\prime}} \cdot(\nabla \Phi)_{i}
$$

que permite realizar avaliações sobre as posições das partículas em relação à interface nos sucessivos passos de simulação. Neste sentido, o resultado final obtido é uma interface bem definida (Fig. 4).

\section{REDISTRIBUIÇÃO DE MASSA EM TÉCNICAS DE CORREÇÃO DE INTERFACE EM MÉTODOS ISPH}

Embora a técnica de correção de interface possua grandes vantagens, a constante alteração de partículas entre as fases pode acabar provocando efeitos indesejados com relação à conservação de massa, podendo gerar erros numéricos durante a simulação. Uma forma de ver isso é através de uma análise sobre a evolução do volume normalizado de uma das fases, definido como:

$$
V_{n}(t)=\frac{V(t)}{V_{0}}
$$

onde $V_{0}$ é o volume inicial da fase, e $V(t)$ o volume no instante $t$.

A Fig. 5 apresenta uma análise da evolução do problema descrito na Fig. 3, onde é possível notar a variação do volume ao longo do tempo. Para resolver este problema pode ser realizada uma redistribuição da massa entre as partículas que representam cada fase do escoamento. Neste sentido, sabe-se que inicialmente, um escoamento bifásico tem associado à ele uma fase $\mathrm{A}$ com massa total $M_{A}$, e uma fase $\mathrm{B}$ com massa total $M_{B}$. Além disso, para que a lei de conservação de massa não seja violada, os valores de $M_{A}$ e $M_{B}$ não devem sofrer alterações, considerando que não há inserções adicionais de matéria no sistema. A partir disso, inicialmente, para cada partícula de fluido é associada uma massa $m_{i}$, tal que:

$$
m_{i}=M_{A} / N_{A}
$$




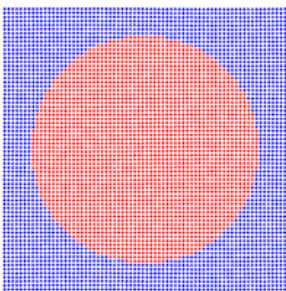

(a)

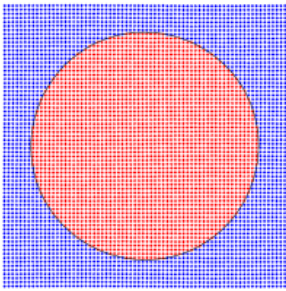

(f)

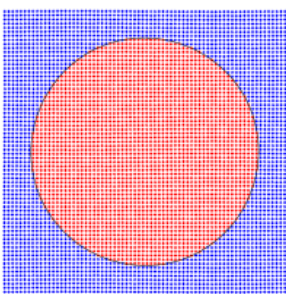

(k)

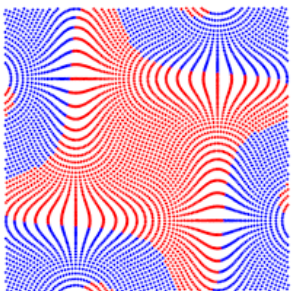

(b)

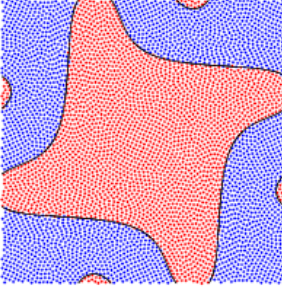

(g)

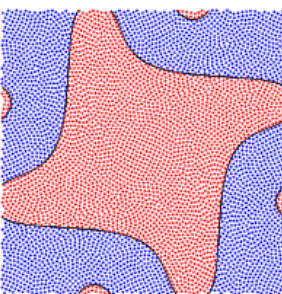

(1)

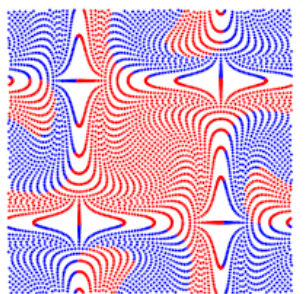

(c)

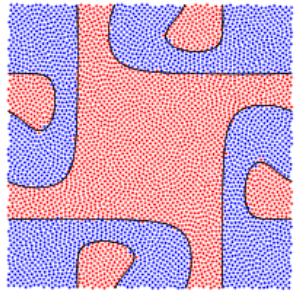

(h)

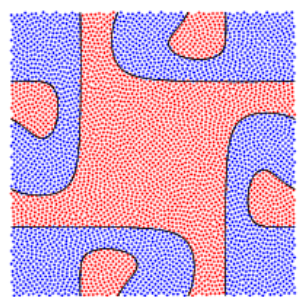

(m)

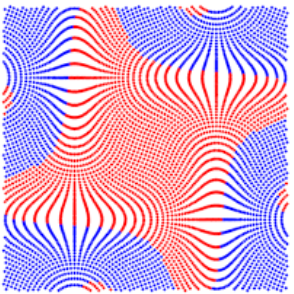

(d)

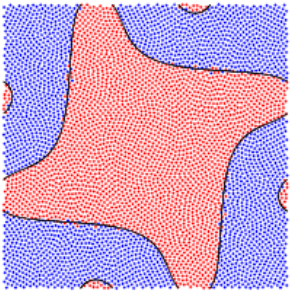

(i)

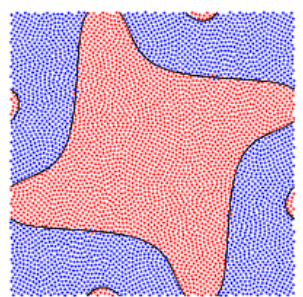

(n)

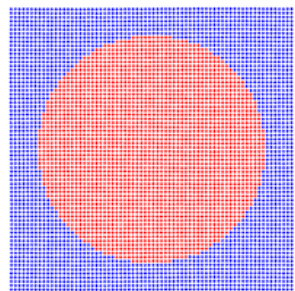

(e)

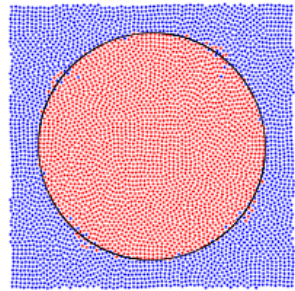

(j)

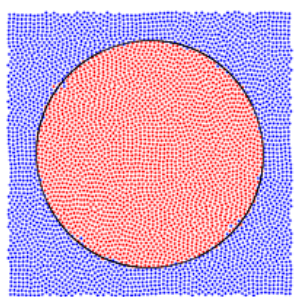

(o)

Figura 3. Integração das trajetórias em um escoamento de Taylor-Green: (a-e) ; (f-j) com correção de Xu et al. (2009); (k-o) com correção de Xu et al. (2009) e correção de interface de Cordeiro et al. (2013). Em cada linha são representados os passos: inicial $(\mathrm{a}, \mathrm{f}, \mathrm{k}), 200$ passos $(\mathrm{b}, \mathrm{g}, \mathrm{l}), 400$ passos (onde ocorre a inversão do campo)(c, $\mathbf{h}, \mathbf{m})$, 600 passos $(d, i, n)$, e (e) configuração final, após 800 passos $(e, j, 0)$.

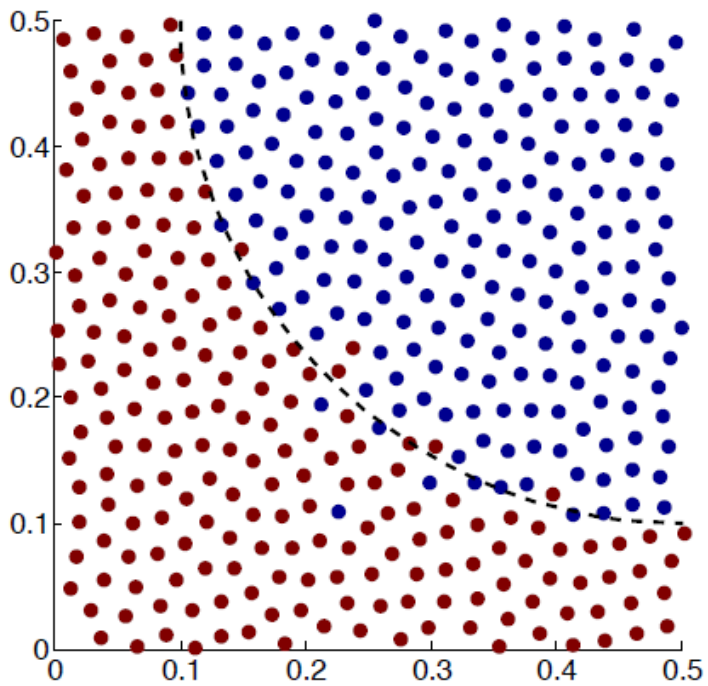

(a)

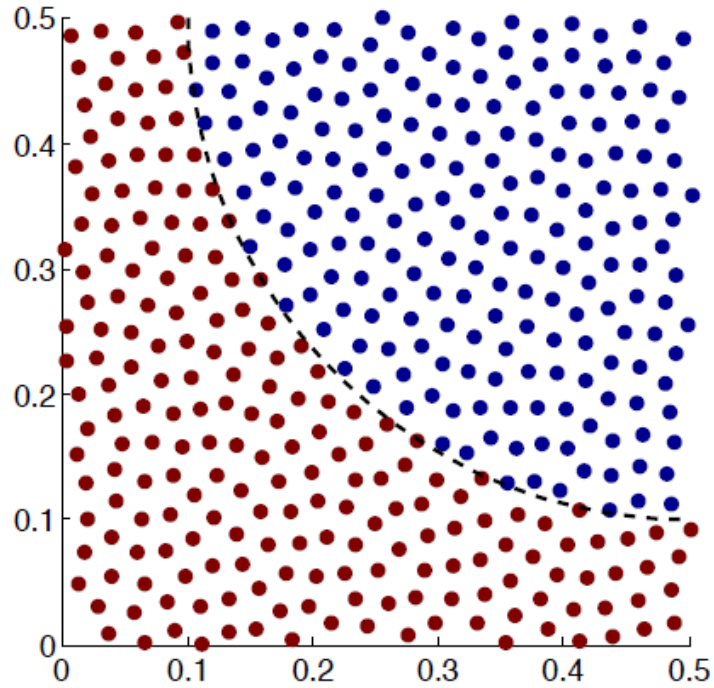

(b)

Figura 4. Detalhe da configuração final obtida: (a) usando correção de Xu et al. (2009), e (b) usando correção de Xu et al. (2009) e correção de interface de Cordeiro et al. (2013).

onde $N_{A}$ é o número de partículas da fase $\mathrm{A}$. Para as partículas da fase $\mathrm{B}$, o cálculo é similar.

Com base nisso, em cada passo de simulação, onde são realizadas alterações das propriedades das partículas proveniente da correção de interface, tem-se uma alteração na quantidade de partículas de cada fase. Neste sentido, realiza-se uma nova redistribuição de massa $m_{i}$ para as partículas, com base na nova quantidade de partículas de cada fase e con- 
siderando os valores $M_{A}$ e $M_{B}$, os quais são constantes durante toda a simulação. Os novos valores de $m_{i}$ podem ser obtidos utilizando-se novamente a equação 25. Essa abordagem irá garantir a conservação do volume ao longo do tempo, como mostra a Fig. 5.

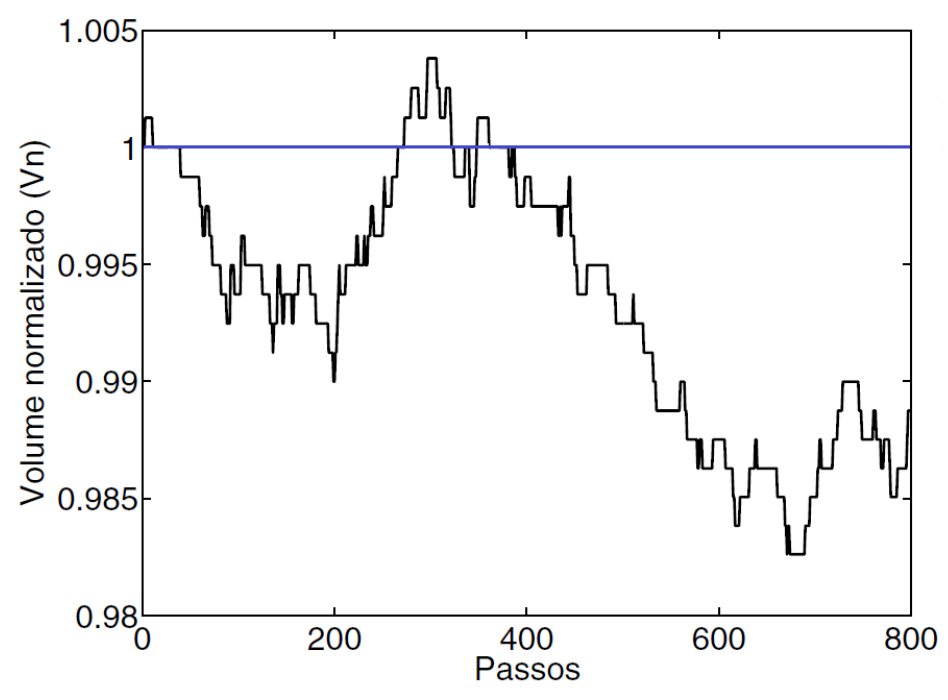

Figura 5. Histórico de conservação de volume para a técnica ISPH com correção de interface: (em negro) sem utilização da técnica de redistribuição de massa, (em azul) com utilização da técnica de redistribuição de massa.

\section{CONCLUSÃO}

Neste trabalho foi apresentado um aperfeiçoamento da técnica de correção de interface baseada em Série de Taylor para métodos ISPH através da inserção de rotinas de redistribuição de massa. Embora a técnica seja relativamente simples, ela representa ganhos consideráveis no que se refere à prevenção de possíveis erros numéricos e a não violação da lei de conservação de massa.

\section{REFERÊNCIAS}

Chorin, A. J. 745-762. Numerical solution of the navier-stokes equations. Mathematics of Computation, 22.

Cleary, P., e Monaghan, J. 1999. Conduction modelling using smoothed particle hydrodynamics. J. Comput. Phys., 148(1), 227-264.

Cordeiro, D. F., Sousa, A., F. S. Castelo Filho, e Nóbrega, J. M. 2013. Uma técnica de correção de interface para o método incompressible smoothed particles hydrodynamics. Trends in Applied and Computational Mathematics, 4(3), $347-358$.

Cummins, S., e M., R. 1999. An sph projection method. Journal of Computational Physics, 152, 584-607.

Gingold, R. A., e Monaghan, J. J. 1977. Smoothed particle hydrodynamics - Theory and application to non-spherical stars. Royal Astronomical Society, 181, 375-389.

Hu, X. Y., e Adams, N. A. 1995. A multi-phase sph method for macroscopic and mesoscopic flow. Journal Comput. Phys, 213(2), 844-861.

Lee, E. S., Moulinec, C., Xu, R., Violeau, D., Laurence, D., e Stansby, P. 2008. Comparisons of weakly compressible and truly incompressible algorithms for the sph mesh free particle method. Journal of Computational Physics, 227.

Liu, G. R., e Liu, M. B. 2003. Smoothed Particle Hydrodynamics - a meshfree particle method. Singapure: World Scientific.

Lucy, L. B. 1977. A numerical approach to the testing of the fission hypothesis. Astron. Journal, 82, 1013-1024.

Marrone, S., Colagrossi, A., Touzé, D. L., e Graziani, G. 2010. Fast free-surface detection and level-set function definition in $\{\mathrm{SPH}\}$ solvers. Journal of Computational Physics, 229(10), $3652-3663$.

Monaghan, J. J. 1994. Simulating free surface flow with sph. Journal of Computational Physics, 110, $399-406$.

Morris, J. P. 1996. Analysis of Smoothed Particle Hydrodynamics with Applications. Phd thesis, Monash University.

Petronetto, F. 2008. A Equação de Poisson e a Decomposição de Helmholtz-Hodge com Operadores SPH. Phd thesis, Pontifícia Universidade Católica do Rio de Janeiro.

Shao, S., e Lo, E. Y. M. 2003. Incompressible sph method for simulating newtonian and non-newtonian flows with a free surface. Advances in Water Resources, 26, 787-800.

$\mathrm{Xu}, \mathrm{R}$., Stansby, P., e Laurence, D. 2009. Accuracy and stability in incompressible sph (isph) based on the projection method and a new approach. Journal of Computational Physics, 228, 6703-6725. 


\title{
RESPONSABILIDADE AUTORAL
}

“Os autores são os únicos responsáveis pelo conteúdo deste trabalho”.

\section{CONSERVATION OF VOLUME FOR INTERFACE TREATMENT TECHNIQUES IN ISPH METHODS}

\author{
Douglas Farias Cordeiro, cordeiro@ufg. $\mathrm{br}^{1}$ \\ Ana Teresa Chaves Leite, aninhagta. chaves@gmail. com ${ }^{2}$ \\ Ciro José Almeida Macedo, ciro.macedo@ifg.edu.br ${ }^{2}$ \\ Marcos Aurélio Batista, marcos@catalao.ufg. $\mathrm{br}^{3}$ \\ ${ }^{3}$ Universidade Federal de Goiás - Faculdade de Informação e Comunicação \\ Campus Samambaia, Goiânia - GO, 74.001-190. \\ ${ }^{4}$ Instituto Federal de Educação, Ciência e Tecnologia de Goiás \\ Campus Cidade de Goiás, Quartel do XX, Praça Brasil Ramos Caidado, Goiás - GO, 76.600-000. \\ ${ }^{5}$ Universidade Federal de Goiás - Departamento de Ciência da Computação \\ Av. Dr. Lamartine P. Avelar, 1120, Catalão - GO, 75.704-020.
}

\begin{abstract}
The Smoothed Particle Hydrodynamics is particle-based method, used for fluid dynamics problems. Among the strategies for implementing the SPH method, the ISPH (Incompressible SPH) has better accuracy and stability of results. The ISPH may be improved using artificial displacement techniques, removing the particles from their natural trajectories. However, this technique causes a unphysical behavior on the interface between the fluids. To prevent this, Cordeiro et al. (2013) introduces a interface treatment technique based on Level Set, which provides considerable results. However, the technique presents some volume variation during the simulations. Here is presented an improvement to the treatment interface technique by incorporating routines that prevent these volume variation.
\end{abstract}

Keywords: sph, numerical methods, conservation of volume, two-phase flows 